\title{
A Primary Pleural Hydatid Cyst in an Unusual Location a Report of Four Cases
}

\author{
Karima Bouytse , Fatima Zahra Mrabet, Hanane Haddaoui, Jamal-Eddine Bourkadi
}

Moulay Youssef Hopital, Pulmonary Unit, University Mohammed V of Rabat, Morocco

DOI: $10.36347 /$ sjmcr.2020.v08i03.040

| Received: 16.03.2020 | Accepted: 23.03.2020 | Published: 27.03.2020

*Corresponding author: Karima Bouytse

Abstract

Case Report

Hydatid disease is caused by the larval stage of taenia Echinococcus, which endemic in the Mediterranean region. Recently, the prevalence of the disease has increased worldwide due to an increase in the frequency of travel and immigration. Cyst hydatid mainly affects the liver and the lungs. Primary pleural hydatid disease is a rare variant of this uncommon disease and is rarely reported in medical literature. Here, we report four cases of pleural hydatid cyst discovered in patients, with a nonspecific clinical presentation. The interest of this article is to clarify the epidemiological, clinical and radiological character of the primary pleural hydatid cyst.

Keywords: Primary Pleural Hydatid Cyst.

Copyright @ 2020: This is an open-access article distributed under the terms of the Creative Commons Attribution license which permits unrestricted use, distribution, and reproduction in any medium for non-commercial use (NonCommercial, or CC-BY-NC) provided the original author and source are credited.

\section{INTRODUCTION}

Hydatid disease is a parasitic zoonosis caused by the larval stage of the tapeworm Echinococcus granulosus, Humans are accidental intermediary hosts in the biological life cycle of the parasite. Hydatid cysts are usually located in the liver and lung. The rates of localization of hydatid disease in different body organs vary in the literature [1]. Pleural localization is extremely rare even in endemic countries and represents only $1.3 \%$ of thoracic locations [2]. Here we present our experience and the diagnostic methods in four patients with primary pleural hydatid cyst.

\section{Case Report}

Case Report (1). Mr. SF, a 30-year-old man, without any notable pathological history, asymptomatic on the respiratory plane.

The chest x-ray (Figure 1) showed a homogeneous oval opacity, well limited, left hilar, and having the internal edge in intimate contact with the left edge of the heart .In this context, a chest CT scan revealed a left anterolateral mediastinal mass with a total parietal calcification measuring $70 \mathrm{~mm}$ in height and $55 \mathrm{~mm}$ in lateral diameter (Figure 2). Echocardiography confirmed the presence of left ventricular extracardiac structure without pericardial effusion. Also, magnetic resonance imaging (MRI) showed a mediastinal cyst next to the anterolateral wall of the left cardiac ventricle, in close contact with the pericardium but with a cleavage plane and no mass effect on the cardiac cavities, measuring $72 \mathrm{~mm} * 53 \mathrm{~mm}$. Its tonality was hypointense on $\mathrm{T} 1$ and hyperintense on T2 (Figure 3).

The blood count was normal and the ELISA and Indirect Agglutination serologies were negative. Abdominal sonography showed a normal liver without any cysts or masses in the liver or spleen, hence the primitive character of pleural hydatidosis in this observation. In operation, the heart was of normal volume with no intrapericardial mass. At the opening of the left pleura, the exploration found a solid mass contiguous to the mediastinal pleura and in contact with the left phrenic nerve. The careful dissection and excision of the mass were successfully performed without complications.

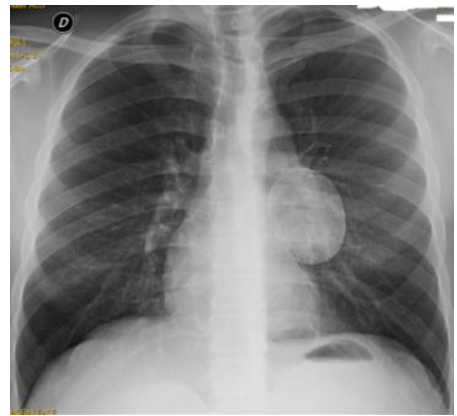

Fig-1: The chest $x$-ray showed a homogeneous oval opacity, well limited, left hilar 

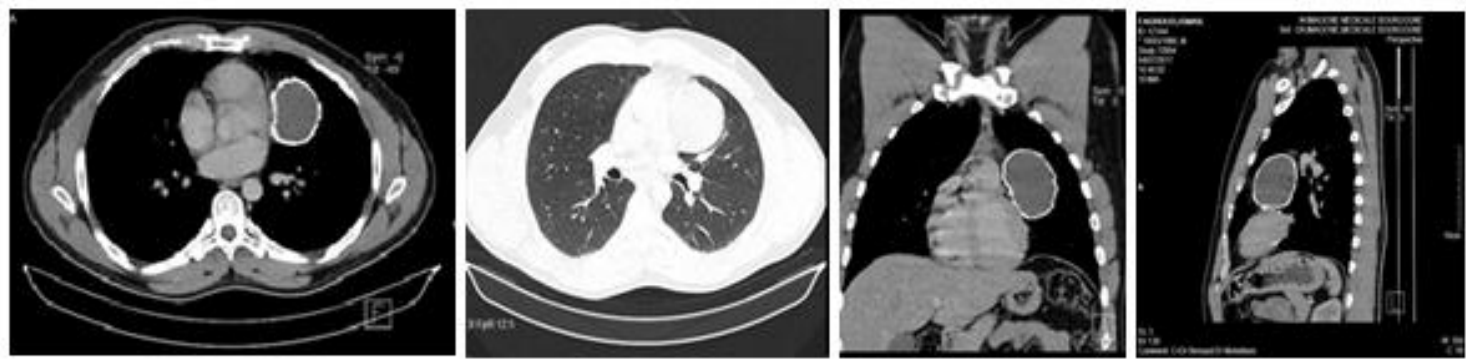

Fig-2: CT scan elucidating a mass with a total parietal calcification (patient 1)

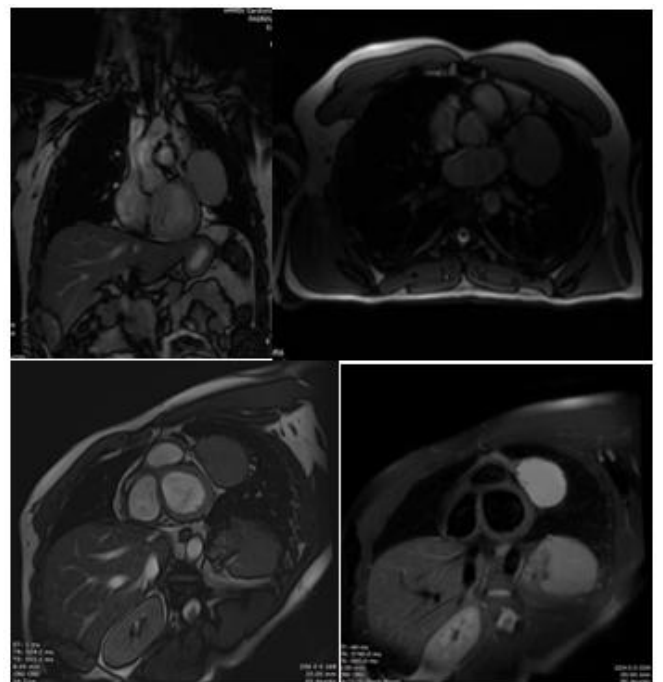

Fig-3: magnetic resonance imaging showed a mediastinal cyst next to the anterolateral wall of the left cardiac ventricle, in close contact with the pericardium (patient 1 )

Case Report (2).Miss M. L., a 20-year-old girl, with no pathological history except a notion of contact with dogs, reported since 3 months right thoracic pain, stage III of mMRC dyspnea, and some episodes of hemoptysis .The clinical examination revealed a right fluid effusion syndrome. The chest ultrasound showed an intrapleural cyst with membranes without associated pleurisy (Figure 4).

The chest scanner revealed a right basothoracic cyst, measuring $126 \times 93 \times 93 \mathrm{~mm}$, with a thickened wall and which enhances after injection of contrast product. In favor of diagnosis of a type II right pleural cyst of Gharbi classification (Figure 5).

The blood count was normal and the ELISA and indirect agglutination serologies were negative. As well, the search for other locations of the hydatid cyst was negative, hence the primitive nature of the pleural hydatidosis in our observation. During surgery, the presence of a cystic formation in the parietal pleura was noted. The delicate dissection had objectified thickened visceral pleura. The cystectomy was successfully performed and the part was sent to the parasitology laboratory whose presence of scolex living in the intracystic fluid was noted.

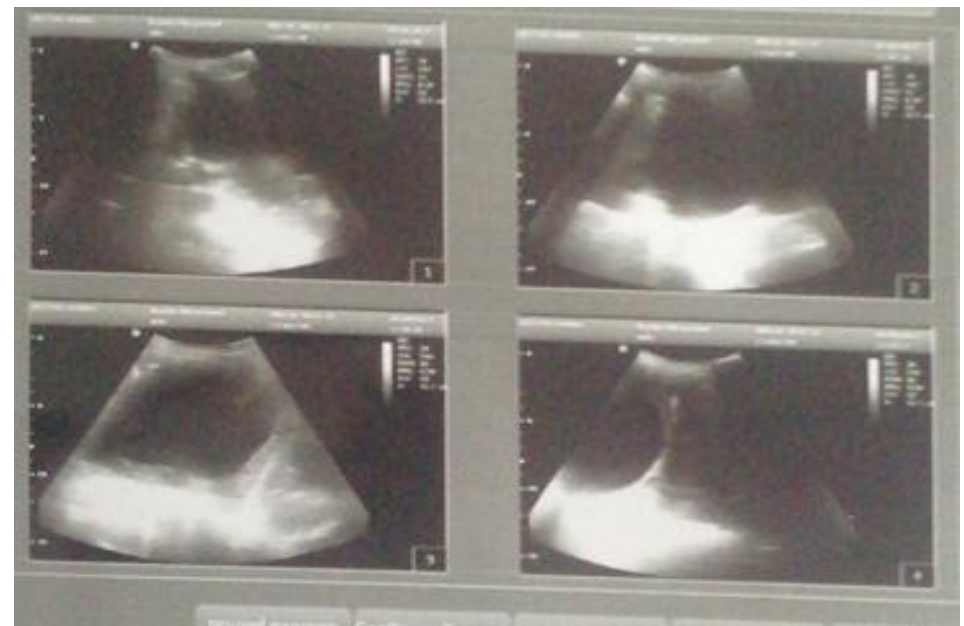

Fig-4: The chest ultrasound showed an intrapleural cyst with membranes (patient 2) 


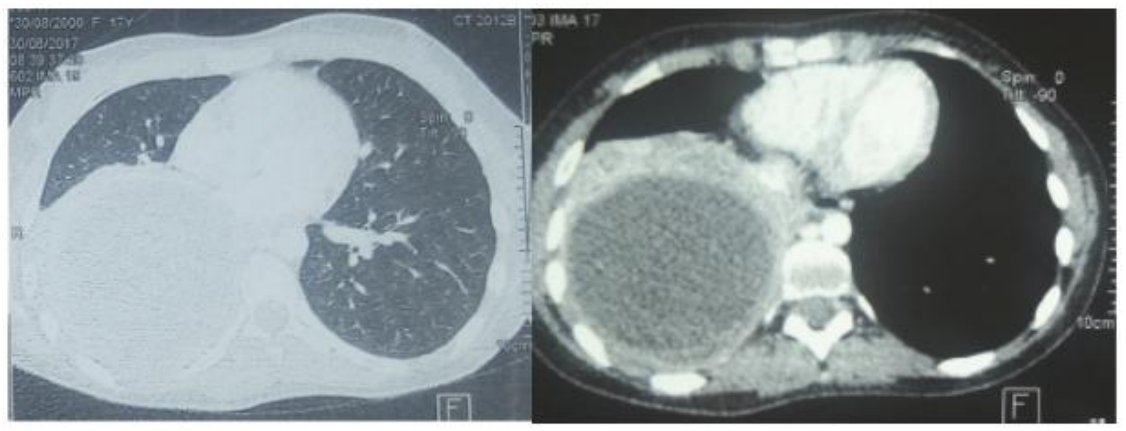

Fig-5: Chest scanner showed a right basothoracic cyst (patient 2)

Case Report (3). A.Y 59 years old, with no particular pathological history who presented for 6 months left chest pain with cough, purulent expectoration and dyspnea stage II of the mMrc. The clinical examination the chest was moving symmetrically; there was no hyper resonance or dullness, and air entry was bilaterally equal.

Chest radiography showed heterogeneous opacity of calcified contours right paracardiac, with left basal opacity of water tone that effaced the left cul de sac (figure 6).

The thoracic CT has objectified a right paracardiac cystic formation of $57 * 45 \mathrm{~cm}$ well limited, seat of fine arcuate peripheral calcification, its content is heterogeneous not enhancing after contrast with liquid formation of upper pericardial recess behind the ascending aorta measuring $25 \mathrm{~mm}$ (figure 7).

The biological tests were normal (blood count; sputum culture for tuberculosis (TB)) except the hydatic serology (ELISA) which was positive abdominal ultrasound and brain scan were normal. The patient is lost to follow-up and therefore the investigations have not been completed

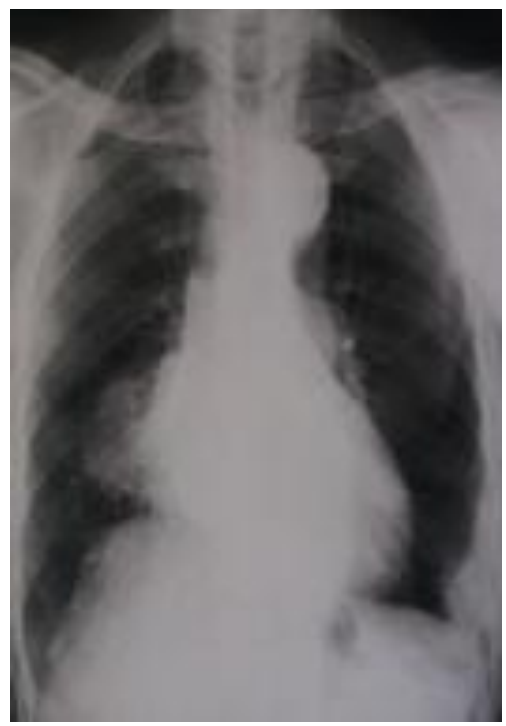

Fig-6: Chest radiography showed heterogeneous opacity of calcified contours right paracardiac, with left basal opacity of water tone that effaced the left cul de sac (patient 3 ).
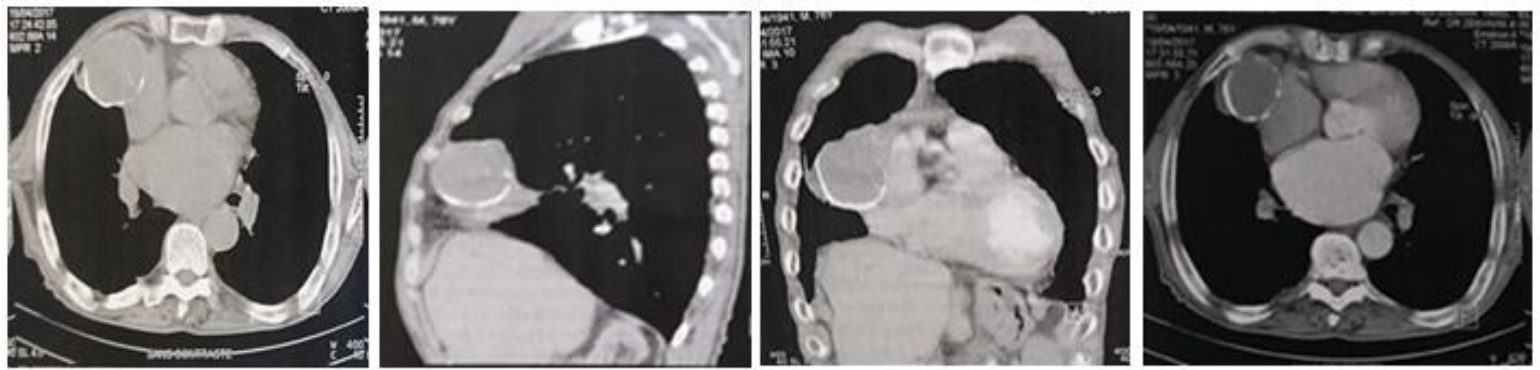

Fig-7: The thoracic CT objectified a right paracardiac cystic formation well limited, seat of fine arcuate peripherical calcification with liquid formation of upper pericardial recess behind the ascending aorta (patient 3)

Case Report (4).A.B ; A 62-year-old men, farmer living in a sheep-raising area, without notable history, who for 2 months presented a cough bringing back purulent sputum with appearance 7 days ago of left chest pain in side stitch. Clinical examination showed mixed effusion syndrome on the left.

Chest X-ray showed homogeneous left basal opacity that effaced the diaphragmatic cupola, topped by avascular hyperclarity (left hydro pneumothorax).
The patient benefited from an axillary thoracic drainage at the 4th intercostal space. Chest CT after drainage revealed a cystic formation with thickened and regular wall, it exerts a mass effect on the underlying parenchyma which is collapsed and it measures $3.4 *$ $6.5 * 7.5 \mathrm{~cm}$ (figure 8 ).

Since it was most probably a hydatid cyst, surgical management was preceded on the left by a posterolateral thoracotomy in the sixth intercostal space, the pleural symphysis was opposite the lesion 
and the pleural pocket was opened containing a hydatid membrane bathing in a liquid "rock water". An extraction of the membrane and abundant washing of the pleural cavity with hypertonic saline solution and oxygenated water was done, a few minutes later it was aspirated completely and directly toward the aspirator.
After that, it was mobilized, dissected, and removed completely en-bloc, then a pleural decortication was performed, continuous suction chest drainage was left in the pleural cavity, and the thoracotomy was closed after that (figure 9).
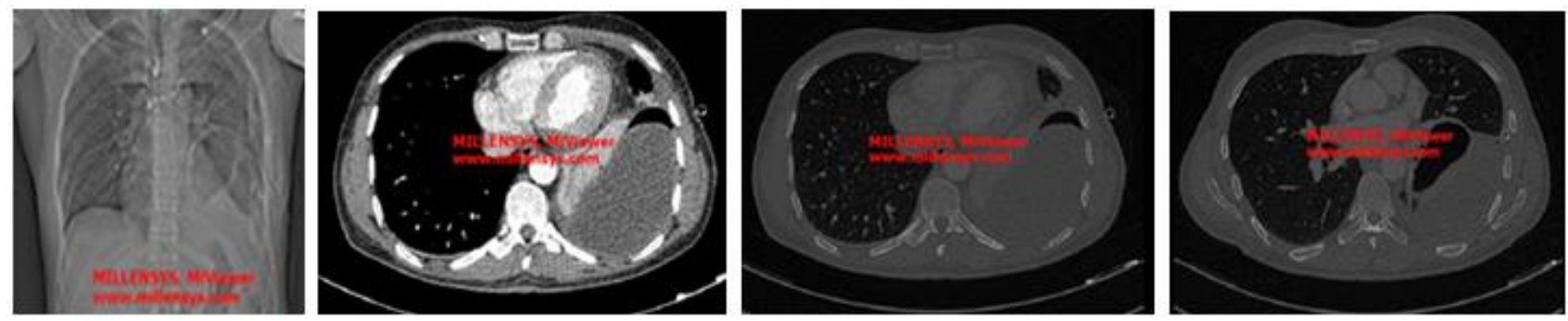

Fig-8: Chest CT revealed a cystic formation with thickened and regular wall, exercising a mass effect on the underlying parenchyma (patient 4)
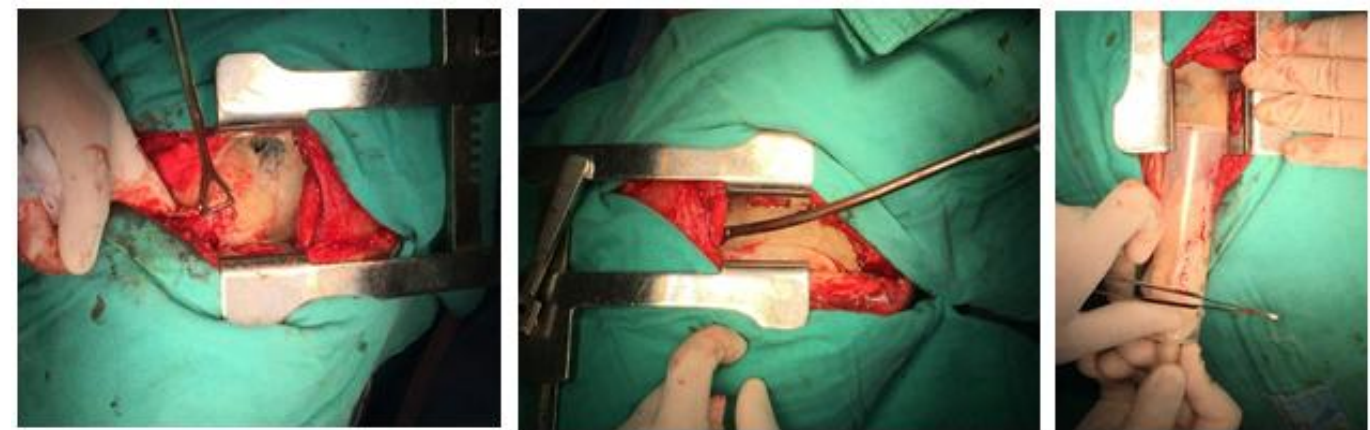

Fig-9: Intraoperative view: Cystic lesion that bulges through intercostal space.

\section{DISCUSSION}

Hydatid cyst disease is a zoonotic disease caused by the larval stage of Echinococcus granulosus (dog tapeworm), E. multilocularis, or E. vogeli [3]. This disease occurs when humans ingest the hexacanth embryos of the dog tapeworm. Infestation by hydatid disease in humans most commonly occurs in the liver (55-70\%) followed by the lung (18-35\%); the two organs can be affected simultaneously in about $5-13 \%$ of cases [4]. intrathoracic but extrapulmonary locations like the pleura (representing only 1.3\%), diaphragm, mediastinum, pericardium, and chest wall are uncommon [5]. Pleural hydatid cysts can develop chiefly as a result of liver or lung cyst rupture into the pleural space with complications of pneumothorax, pleural effusion or empyema [6]. However, a hydatid cyst located primarily in the pleural space, as observed in our cases, is very rare. Only about $15 \%$ of larvae succeed in passing through the hepatic and pulmonary capillary barrier to reach the systemic circulation [7] Hydatid cysts consequently can be found in any tissue but more vascularized tissues have a greater chance of implantation. Because pleural membranes have only small blood capillary networks, it is difficult for the cysts to reach the pleural space through the bloodstream [5]. It is uncommon even in an endemic country that hydatid disease directly invading the pleura without hepatic or pulmonary involvement.
Most patients with hydatid disease present with a variety of symptoms including cough, chest pain, hemoptysis, malaise, fever and even expectoration of cystic materials, but others can be asymptomatic like our first patient. Intrathoracic extrapulmonary cysts may produce compression symptoms in surrounding vital structures [8].

Biological diagnosis of hydatidosis based on blood count wich may reveal hyper-eosinophilia (> 500 / $\mathrm{mm} 3$ ), and a different immunoassays which are often negative in cysts uncomplicated isolated pleura. The sensibility immunology clearly increases in case of associated complication or hepatic cyst [9]. we have three out of four patients had negative immunologic tests similar to that described in the literature.

Imagery is a fundamental element for the diagnosis positive and for follow-up. The radiography of the chest shows a tonic pleural opacity homogeneous well limited with water tonality. Rarely, it shows peripheral calcifications allowing guiding the diagnosis. Ultrasound, second-line examination, allows to confirm the fluid nature of pleural opacity and make a positive diagnosis, particularly in the case of a multivesicular form.and In many cases, it shows the proliferative membrane doubling inside the pericyst which is pathognomonic of hydatid cyst, it also makes it possible to detect a possible pleural effusion associated with it and to look for other cystic localizations, particularly 
abdominal ones [2]. Computed tomography confirms the pleural localization of the well limited fluid unmodified by IV injection of contrast. CT also has the advantage to be superior to the chest $\mathrm{x}$-ray by detecting the peripheral calcifications fines [2]. MRI allows bettering specifying the topography of the cyst and its contact with underlying organs thanks to multiplanar sections. The T2-weighted sequences visualize small daughter vesicles or a floatingmembrane in favor of the diagnosis and especially a peripheral $\mathrm{T} 1$ and $\mathrm{T} 2$ hyposignal in relation to the calcium deposits which is pathognomonic to the diagnosis [10].

Surgery is the treatment of choice by cystectomy. As a general rule, when the presurgical diagnosis of hydatid cyst is suspected, surgeon should take care of four things in order to achieve complete resection and to avoid recurrence of disease from pleural hydatid cysts:

1) Planning the appropriate surgical approach to prevent cystic rupture or spillage when doing the thoracotomy;

2) inactivation of daughter cysts and scolices prior to removal by injecting $20 \%$ hypertonic saline solutions into thecyst;

3) no spillage of cyst contents during surgery to avoid anaphylactoid reaction, recurrence, and multiple hydatidosis

4) complete removal of the cyst including the innermost germinative layer, which can produce scolices, with en-bloc excision whenever possible; sometimes, to avoid recurrence, it is necessary to respect the affected surrounding tissues completely; postoperatively, it may be necessary to place patients on an anthelmintic medical regimen (Albendazol) with appropriate follow-up reevaluations [11].

\section{CONCLUSION}

Primary pleural localization of the cyst hydatic is very rare or even exceptional. Her discovery is often fortuitous. More rarely, this form of hydatid disease is symptomatic with a clinical symptomatology nonspecific. The imagery in particular in section, plays a fundamental role. Indeed, it allows evoking the diagnosis, to specify the topography and relations with underlying organs, and look for other locations.

\section{REFERENCES}

1. Geramizadeh B.Unusual locations of the hydatid cyst: a review from iran. Iran J Med Sci. 2013;38(1):2-14.

2. Badji NF, NDong B, Akpo G, Dème H, Touré MH, Niang El H. Apport De L'Imagerie Dans Le Diagnostic De Kyste Hydatique Pleural Primitif: à Propos De Deux Cas. Mali Médical. 2017 Oct $1 ; 32(4)$.

3. Srinivasan B, Mohite PN, Thingnam SK. Extrapulmonary intrapleural hydatid cysts-rare variant of uncommon disease. Indian Journal of Thoracic and Cardiovascular Surgery. 2010 Dec $1 ; 26(4): 247-50$.

4. Saidi F. Treatment of echinococcal cysts. In: Nyhus LM, Baker RJ, Sabiston DC, editors. Mastery of surgery. 2nd ed. London: Little, Brown and Company. 1992: 818-37.

5. Gursoy S, Ucvet A, Tozum H, Erbaycu AE, Kul C, Basok O. Primary intrathoracic extrapulmonary hydatid cysts: analysis of 14 patients with a rare clinical entity. Tex Heart Inst J. 2009; 36:230-3.

6. Turgut AT, Altinok T, Topçu S, Koşar U. Local complications of hydatid disease involving thoracic cavity: imaging findings. Eur J Radiol. 2009;70:4956.

7. Kim SJ, Jung KH, Jo WM, Kim YS, Shin C, Kim JH. A case of pleural hydatid cyst mimicking malignancy in a non-endemic country. Tuberc Respir Dis. 2011;70:338-341.

8. Gürsoy S, Ucvet A, Tozum H, Erbaycu A.E, Kul C, Basok O. Primary intrathoracic extrapulmonary hydatid cysts. Texas Heart Institute Journal. 2009; 36(3): 230-233. PMid: 19568393.

9. Nazaroglu H, Balci A, Bukte Y, Simsek M. Giant intrathoracic extrapulmonary hydatid cyst manifested as unilateral pectus carinatum. South Med J. 2002, 95, 1207-1208.

10. Harzallah L, Bacha M, Garrouche A, Messak A, Ben Cherifa L. Kyste hydatique pleural primitif: a propos d'une observation. RMLG. Revue médicale de Liège. 2007;62(7-8):506-8.

11. Alakhras Aljanadi F. A rare case of primary hydatidosis; A primary parietal pleural hydatid cyst. Acta Health Medica. 2016; 1 (4) : 93-97. 